\title{
Trombosi tardiva della vena del rene trapiantato: descrizione di un caso
}

\author{
P. L. Bedani ${ }^{1}$, M. Bergami ${ }^{1}$, G. Mugnani ${ }^{2}$, R. Galeotti ${ }^{3}$ \\ ${ }^{1}$ Divisione di Nefrologia, Azienda Ospedaliera S. Anna, Ferrara \\ ${ }^{2}$ Scuola di Specializzazione in Nefrologia \\ ${ }^{3}$ Istituto di Radiologia, Università degli Studi di Ferrara, Ferrara
}

a trombosi della vena renale è solitamente una complicanza precoce del trapianto renale. $\mathrm{La}$ sua incidenza nel periodo immediatamente post-intervento oscilla tra l'1 e il $4 \%$ ed è dovuta principalmente a complicanze chirurgiche $(1,2)$.

In fase tardiva, dopo anni di trapianto ben funzionante, l'evenienza di tale complicanza è rara $(3,4)$ ma per la difficoltà di un efficace trattamento medico rappresenta un rischio reale di perdita dell'organo trapiantato.

In questo lavoro gli autori descrivono un caso di trombosi combinata della vena iliaca e della vena renale in una paziente trapiantata da oltre quattro anni che ha risposto favorevolmente al trattamento trombolitico loco-regionale e sistemico.

\section{Caso clinico}

Una paziente di 61 anni, giunta all'uremia terminale per polichistosi renale bilaterale, era stata sottoposta a trapianto renale da cadavere, posizionato in fossa iliaca sinistra, all'età di 57 anni. Il trattamento immunosoppressivo prevedeva l'assunzione di ciclosporina, prednisone e azatiopri- na. Il decorso clinico era stato caratterizzato da un rigetto acuto nel primo mese post-trapianto e da alcuni episodi di tachicardia parossistica sopraventricolare negli anni successivi. La funzionalità renale, dopo l'episodio di rigetto, si era stabilizzata su valori di creatininemia pari o inferiori a $1.5 \mathrm{mg} / \mathrm{dl}$. L'esame delle urine non aveva mai evidenziato proteinuria. Era presente da anni ipercolesterolemia $(280 \mathrm{mg} / \mathrm{dl})$. Per la comparsa di lieve edema all'arto inferiore sinistro e dolenzia in sede sovrainguinale omolaterale la paziente fu visitata ambulatorialmente, senza tuttavia un orientamento diagnostico-terapeutico mirato. Qualche giorno più tardi il quadro edemigeno si aggravò inducendo la paziente al ricovero ospedaliero. L' ecocolor doppler evidenziò una estesa trombizzazione della vena iliaca esterna di sinistra con sospetta occlusione parziale della vena del rene trapiantato omolateralmente. La paziente fu pertanto sottoposta a studio angio-TC che confermò l'esistenza di una trombosi venosa massiva dell'asse iliaco-femorale con parziale coinvolgimento della vena del rene trapiantato (Fig. 1A) . L'esame TC mostrò inoltre lo schiacciamento contro il corpo vertebrale, per una verosimile malformazione di tipo congenito, della vena iliaca sinistra da parte dell'arteria (Fig. 2). La conta e la funzionalità piastrinica, il tempo di protrombina e di tromboplastina parziale, e i livelli di antitrombina III erano nella norma. Previo posizionamento di un filtro cavale per via transgiugulare destra, la paziente fu sottoposta a trattamento con Eparina sodica ( $24.000 \mathrm{U} / 24$ ore). Nello stesso tempo, tramite catetere transfemorale destro, si avviò l'infusione di Urochinasi $(75.000 \mathrm{U} /$ ora) in vena iliaca sinistra, al di sopra del trombo. Dopo due giorni di terapia, senza evidenti segni di ricanalizzazione venosa, si è provveduto, tramite incannulamento dell'arteria femorale sinistra, allo studio angiografico selettivo del rene trapiantato, che non mostrò alterazioni significative della perfusione dell'organo. Utilizzando il catetere lasciato in arteria renale si predispose l'infusione di Urochinasi (50.000 U/ora), riducendo contemporaneamente sia la somministrazione di Eparina (12.000 U/24 ore) che di Urochinasi per via endovenosa $(50.000 \mathrm{U} /$ ora) e fu rimosso il filtro cavale. L'arteriografia renale di 
Fig. 1- Angio CT del rene trapiantato. A) Normale perfusione arteriosa del rene. Pervietà dell'arteria iliaca sinistra (punta della freccia). Trombosi delle vene iliaca sinistra (freccia diritta) e renale (freccia curva). B) Dopo tre mesi, completa ricanalizzazione della vena iliaca (freccia diritta) e della vena renale (freccia curva).

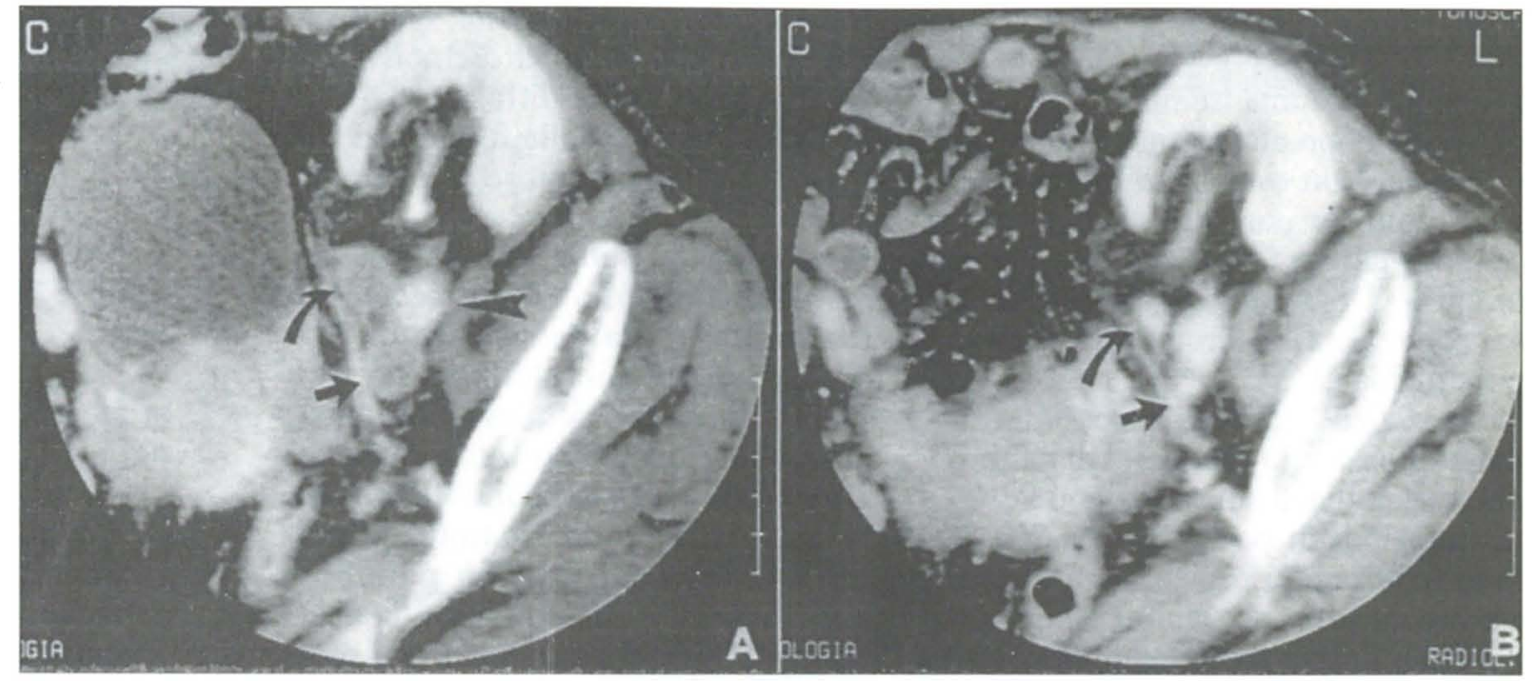

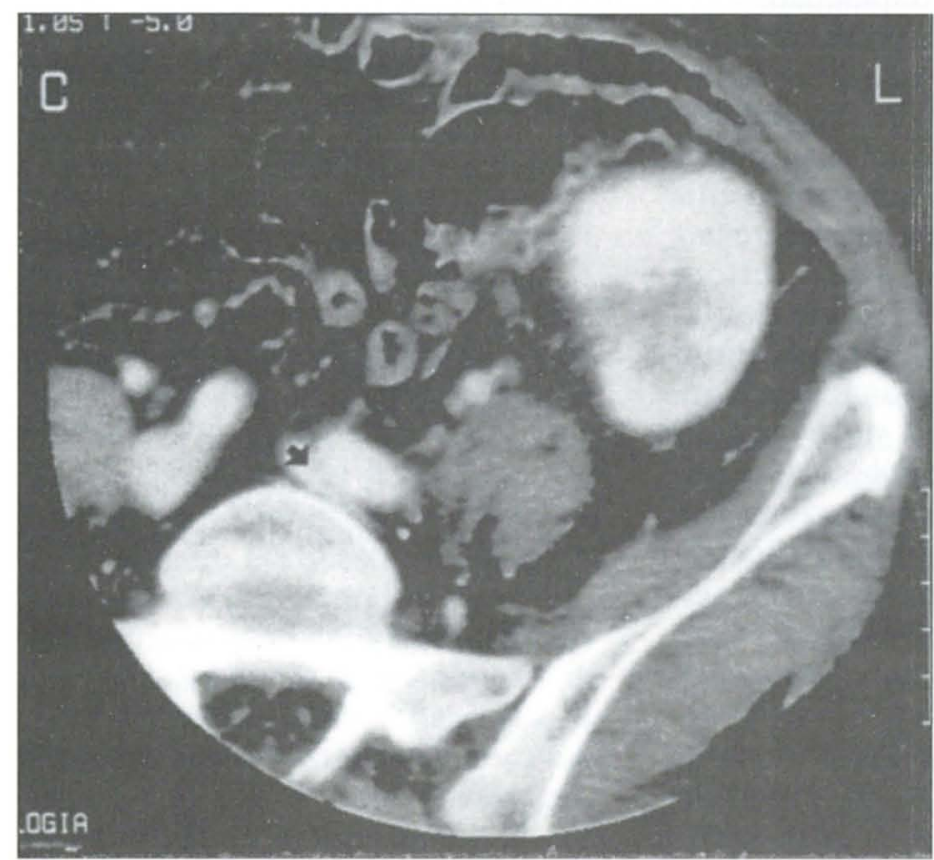

Fig. 2 - Angio CT. Vena iliaca sinistra (freccia) compressa tra il corpo vertebrale e l'arteria iliaca sinistra.

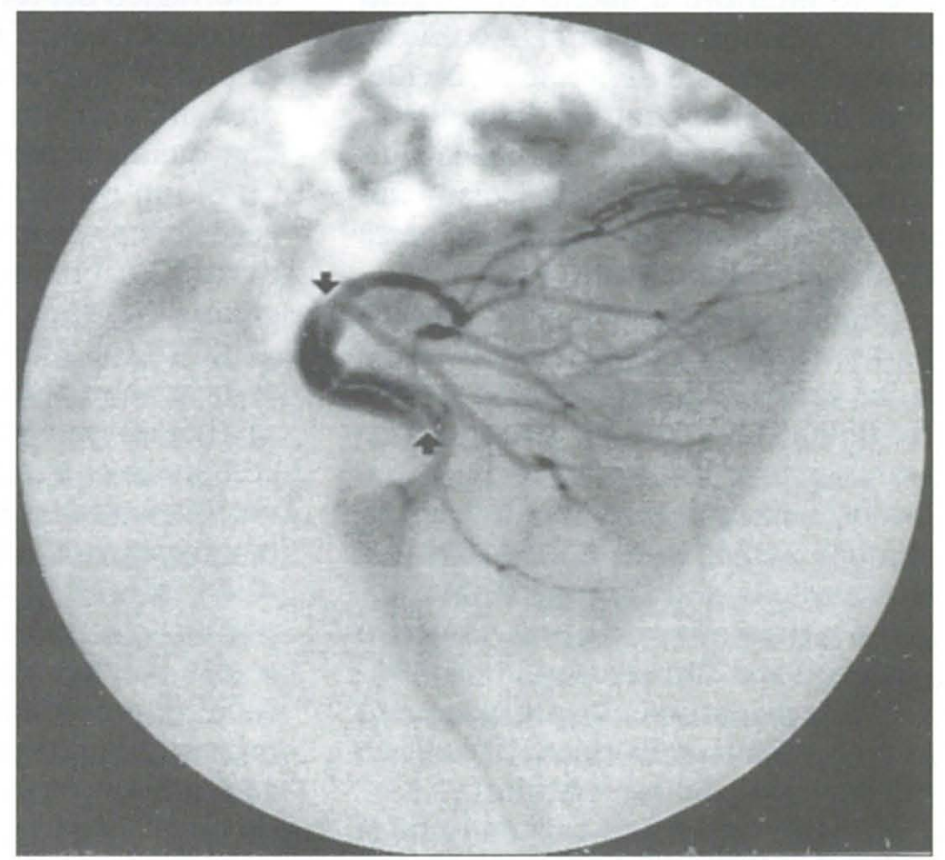

Fig. 3 - Arteriografia renale dopo circa 20 ore di trombolisi regionale. La freccia indica lo slaminamento della parete arteriosa. controllo, eseguita dopo circa 20 ore, mostrò lo slaminamento della parete arteriosa subito a valle dell'anastomosi (Fig. 3). Tale complican$\mathrm{za}$, unitamente al formarsi di un grosso ematoma in sede inguinale sinistra, causa di anemizzazione acuta ( $\mathrm{Hb} 7.5 \mathrm{~g} / \mathrm{dl})$, ci indusse a sospendere la terapia trombolitica e a rimuovere entrambi i cateteri. Dopo alcuni giorni, durante i quali la funzionalità renale presentò una lieve instabilità (creatininemia 1.9 - 2.5 $\mathrm{mg} / \mathrm{dl})$ senza modificazioni signifi- cative della diuresi e in assenza di proteinuria, la paziente venne dimessa con indicazione a proseguire la terapia anticoagulante con Eparina a basso PM (4000 U/die sc) e Acenocumarolo. Dopo circa un mese la terapia eparinica fu sospesa e la paziente riprese progressivamente a camminare senza rilevare un incremento significativo dell'edema all'arto inferiore sinistro. Dopo circa due mesi dall'episodio acuto fu eseguito un esame ecocolor doppler che mostrò completa pervietà del- l'asse venoso iliaco-cavale sinistro e della vena renale; permanevano esiti di trombosi alla radice della vena femorale omolaterale. Un mese più tardi venne ripetuta l'angio-TC che confermò la completa ricanalizzazione dei tratti venosi precedentemente trombizzati (Fig. 1B) con la sola persistenza di segni di parziale trombosi della vena femorale sinistra; venne inoltre confermato un lieve aumento di calibro del tratto di arteria renale precedentemente sede di slaminamento parietale. 


\section{Discussione}

Il riscontro di una trombosi della vena renale dopo alcuni anni di trapianto renale è un'evenienza molto rara, soprattutto in pazienti che non presentano fattori favorenti, quali: proteinuria, glomerulopatia membranosa, con o senza sindrome nefrosica, e policitemia $(3,5)$.

Nella paziente qui segnalata la nefropatia di base e la riduzione di calibro della vena iliaca sinistra sono risultate situazioni di potenziale rischio. È noto infatti che i pazienti con displasia cistica sono più inclini alla trombofilia e hanno un sistema vascolare sia arterioso che venoso di calibro spesso inferiore a quello di soggetti di pari età non policistici. Accanto a questa situazione di per sé favorente, la paziente era portatrice di un'alterazione di calibro della vena iliaca sinistra a causa del suo schiacciamento contro il corpo vertebrale da parte dell'arteria omolaterale (Fig. 2).

La certezza della diagnosi clinica ha suscitato una certa discussione sulla scelta della strategia terapeutica. Poiché la correzione chirurgica risultava controindicata per 1'alto rischio di complicanze connesse all'eventuale tentativo di trombectomia e di rianastomosi della vena renale, abbiamo ritenuto che il trattamento trombolitico farmacologico rappresentasse lo strumento più adeguato per un tentativo di ricanalizzazione del segmento venoso ostruito.

In letteratura non esistono criteri univoci sulla via da utilizzare per la somministrazione di agenti trombolitici in pazienti trapiantati con trombosi della vena renale. Sono state utilizzate sia la via arteriosa (6) che la via venosa $(4,7)$, o entrambe $(8)$. Poiché nella nostra paziente dopo 48 ore di trattamento loco-regionale con Urochinasi per via venosa non si erano colti segni evidenti di ricanalizzazione, fu scelta la somministrazione del farmaco direttamente per via arteriosa. Tale trattamento fu interrotto dopo circa 20 ore per il realizzarsi di alcune complicanze. Si osservò infatti da un lato lo slaminamento della parete dell'arteria renale (Fig. 3), verosimilmente conseguente a micro- trauma indotto dal catetere, e dall'altro si realizzò un quadro emorragico caratterizzato dalla diffusione di petecchie cutanee e dalla formazione di un grosso ematoma in sede inguinale sinistra.

Il favorevole risultato a distanza (ricanalizzazione dei segmenti venosi trombizzati) e la persistenza di una buona funzionalità dell'organo trapiantato ci hanno permesso di ipotizzare che l'infusione intrarenale di Urochinasi abbia impedito l'estensione massiva del processo trombotico a tutta la vena renale. Ciò potrebbe giustificare il mancato rilievo di alcune complicanze attese quali una proteinuria elevata o l'infarcimento emorragico del rene trapiantato.

In conclusione, l'infusione combinata artero-venosa di Urochinasi rappresenta a nostro avviso, in accordo con quanto riportato da altri autori $(3,6)$, un protocollo terapeutico efficace anche se l'effetto può essere tardivo. Ciò che non appare tuttora chiaro è tuttavia il dosaggio dell'agente trombolitico e la necessità di associazione con altri anticoagulanti, al fine di prevenire sia eventuali complicanze emorragiche sia recidive trombotiche. L'associazione con eparina in fase iniziale e con dicumarolici in fase successiva sembra opportuna per garantire l'effetto a distanza dell'agente trombolitico. Il rischio di episodi emorragici con anemizzazione severa, come si è verificato nella nostra paziente, o di complicanze conseguenti al posizionamento di cannule per infusione, non controindicano la scelta di un trattamento acuto di tipo invasivo.

\section{BIBLIOGRAFIA}

1. Jordan ML, Cook GT, Cardella CJ. Ten years of experience with vascular complications in renal transplantation. J Urol 1982; 128: 689692.

2. Merion RM, Calne RY. Allograft renal vein thrombosis. Transplant Proc 1985; 17: 1746-1750.

3. du Buf-Vereijken PWG, Hilbrands LB, Wetzels JFM. Partial renal vein thrombosis in a kidney transplant: management by streptokinase and heparin. Nephrol Dial Tranplant 1998; 13: 499-502.

4. Killewich LA, Pais SO, Sandager G, Flinn WR, Bartlett ST. Salvage of renal allograft function and lower extremity venous patency with thrombolytic therapy: case report and review of the literature. J Vasc Surg 1995; 21: 691-696.

5. First MR, Mendoza N, Maryniak RK, Weiss MA. Membranous glomerulopathy following kidney transplantation. Association with renal vein thrombosis in two of nine cases. Transplantation 1984; 38: 603-7.

6. Chiu AS, Landsberg DN. Successful treatment of acute transplant renal vein thrombosis with selective streptokinase infusion. Transplant Proc 1991; 23: 2297-300.

7. Schwieger J, Reiss R, Cohen JL, Adler L, Makoff D. Acute renal allograft dysfunction in the setting of deep venous thrombosis: a case of seccessful urokinase thrombolysis and a review of the literature. Am J Kidney Dis 1993; 22: 345350.

8. Modrall JG, Teitelbaum GP, Diaz Luna H, Berne T. Local thrombolysis in a renal allograft threatened by renal vein thrombosis. Transplantation 1993; 56: 1011-3. 